\title{
Infinitely Many Periodic Solutions to Delay Differential Equations via Critical Point Theory
}

\author{
Xiaosheng Zhang ${ }^{1}$ and Duo Wang ${ }^{2,3}$ \\ ${ }^{1}$ School of Mathematical Sciences, Capital Normal University, Beijing 100048, China \\ ${ }^{2}$ School of Mathematical Sciences, Peking University, Beijing 100048, China \\ ${ }^{3}$ School of Mathematical Sciences, South China Normal University, Guangzhou 510631, China \\ Correspondence should be addressed to Xiaosheng Zhang; zhangxs@cnu.edu.cn
}

Received 9 June 2013; Revised 8 November 2013; Accepted 14 November 2013

Academic Editor: Yuriy Rogovchenko

Copyright (C) 2013 X. Zhang and D. Wang. This is an open access article distributed under the Creative Commons Attribution License, which permits unrestricted use, distribution, and reproduction in any medium, provided the original work is properly cited.

By the critical point theory, infinitely many $4 \sigma$-periodic solutions are obtained for the system of delay differential equations $\dot{x}(t)=$ $-f(x(t-\sigma))$, where $\sigma \in(0,+\infty)$ and $f \in C\left(\mathbb{R}^{n}, \mathbb{R}^{n}\right)$. It is shown that all the periodic solutions derived here are brought about by the time delay.

\section{Introduction}

This paper is concerned with the existence of periodic solutions to the system of delay differential equations

$$
\dot{x}(t)=-f(x(t-\sigma)),
$$

where $\sigma \in(0,+\infty)$ and $f \in C\left(\mathbb{R}^{n}, \mathbb{R}^{n}\right)$.

Delay differential equations have widely been applied to describe the dynamics phenomena in both natural and manmade processes such as chemistry, physics, engineering, and economics. The existence of the periodic solutions for delay differential equations has been extensively investigated by using various methods, including fixed point theorems [1-5], Hopf bifurcation theorems [6-8], variational methods [9-14], the methods of differential inequalities [15-21], and other effective approaches (e.g., see [22-24]). In [25-31], the minimal periods of the periodic solutions to Lipschitzian differential equations are estimated through the Lipschitz constants (see Remark 4).

The use of variational methods in the study of $4 \sigma$-periodic solutions of system (1) having a variational structure was introduced in 2005 by Guo and $\mathrm{Yu}$ [9]. Assume that

$\left(\mathrm{F}_{1}\right) f$ is odd in $x$; that is, $f(-x)=-f(x)$, for all $x \in \mathbb{R}^{n}$;

$\left(\mathrm{F}_{2}\right)$ there exists $F \in C^{1}\left(\mathbb{R}^{n}, \mathbb{R}\right)$ such that $F_{x}(x)=f(x)$, for all $x \in \mathbb{R}^{n}$, where $F_{x}$ denotes the gradient of $F$.
In [9], the authors obtained the multiplicity results for periodic solutions to (1) in the case that $f$ is asymptotically linear. Later, the existence of the periodic solution of (1) was investigated by using Morse theory and Galerkin methods [10]. For the other relative investigations, we refer the reader to [11-14].

Many practical problems, such as nonlinear population growth models and control systems working with potentially explosive chemical reactions, can be transformed into the form of (1). For example, by the change of variables $y=$ $a \tanh (a x)$, the following generalized food-limited population model

$$
\dot{y}(t)=-\theta \operatorname{sign}(y(t-1))|y(t-1)|^{\gamma}\left(a^{2}-y^{2}(t)\right)
$$

is transformed equivalently into (1) with $n=1, f(x)=$ $\theta a^{\gamma} \operatorname{sign}(x)|\tanh (a x)|^{\gamma}$, and $\sigma=1$, where $\theta$ and $a$ are positive numbers. When $\gamma=1, f^{\prime}(0)=\theta a^{2}$. It is known from [24] that, with the slope $f^{\prime}(0)$ increasing and tending to infinity, the number of the periodic solutions of (2) increases and tends to infinity. Naturally, one would conjecture that when $0<\gamma<1$, (2) possesses infinitely many periodic solutions, since in this case $\lim _{x \rightarrow 0} f^{\prime}(x)=+\infty$.

Motivated by the above observation, in this paper, we study the existence of infinitely many periodic solutions to the system (1) under the assumptions $\left(\mathrm{F}_{1}\right),\left(\mathrm{F}_{2}\right)$, and 
$\left(\mathrm{F}_{3}\right)$ there are $1<\alpha, \beta<2$ and $d_{1}, r_{0}>0$ such that

(i) $0<(f(x), x) \leqslant \alpha F(x)$, for all $x \in B_{r_{0}} \backslash\{\mathbf{0}\}$;

(ii) $d_{1}|f(x)|^{\beta^{\prime}} \leqslant F(x)$, for all $x \in B_{r_{0}}$,

where $1 / \beta+1 / \beta^{\prime}=1, B_{r_{0}}=\left\{x \in \mathbb{R}^{n}:|x| \leqslant r_{0}\right\}$.

Here and subsequently, $(\cdot, \cdot),|\cdot|$ denote the inner product and the standard norm in $\mathbb{R}^{n}$, respectively, and the bold face $\mathbf{0}$ represents the coordinate origin of $\mathbf{R}^{n}$. The main result of this paper is stated as follows.

Theorem 1. Assume that $\left(F_{1}\right)-\left(F_{3}\right)$ hold. Then (1) possesses a sequence of nonconstant $4 \sigma$-periodic solutions $\left\{x_{m}\right\}$ satisfying $\left\|x_{m}\right\|_{\infty} \rightarrow 0$ as $m \rightarrow \infty$

Example 2. When $0<\gamma<1$, it is easy to check that $f(x)=$ $\theta a \operatorname{sign}(x)|\tanh (a x)|^{\gamma}$ satisfies $\left(\mathrm{F}_{1}\right)-\left(\mathrm{F}_{3}\right)$ with $\alpha=\beta=1+\gamma$; then (2) has a sequence of nonconstant 4-periodic solutions $\left\{x_{m}\right\}$ satisfying $\left\|x_{m}\right\|_{\infty} \rightarrow 0$ as $m \rightarrow \infty$.

Remark 3. Let us compare the result here with that in the case of ordinary differential equations (ODE). Without the time delay, (1) reduces to the following system of ODE

$$
x^{\prime}(t)=f(x(t)) .
$$

Let $x(t)=x\left(t ; x_{0}\right)$ be the solution of (3) satisfying the initial condition $x(0)=x_{0} \neq \mathbf{0}$. Then the derivative of the Lyapunov function $V(x)=|x|^{2}$ along $x(t)$ reads

$$
\left.\frac{d V}{d t}\right|_{(3)}=(-f(x(t)), x(t))
$$

From $\left(\mathrm{F}_{3}\right)-(\mathrm{i})$, we see that $d V /\left.d t\right|_{(3)}<0$ for $0<|x|<r_{0}$, which implies that there is no any periodic orbit of (3) across $B_{r_{0}} \backslash\{\mathbf{0}\}$; that is, the trivial solution is an isolated periodic solution. However, by the above theorem, with the time delay, the system (1) possesses infinitely many periodic solutions in any neighborhood of the origin.

Remark 4. Consider the following system of $m$ th order functional differential equations:

$$
x^{(m)}(t)=f(x(\tau(t))), \quad t \in \mathbb{R}
$$

where $f: \mathbb{R}^{n} \mapsto \mathbb{R}^{n}$ satisfies the Lipschitz condition and $\tau: \mathbb{R}^{1} \mapsto \mathbb{R}^{1}$ is a measurable function. The lower bounds for the periods of the periodic solutions to (5) and their special forms are estimated in [25-31]. From this perspective, Theorem 1 complements the information in the case of nonLipschitzian differential equations. For the unique solvability of the periodic problems on functional differential equations, we refer the reader to $[1,15-21]$.

The remainder of this paper is divided into two parts. In the next section, we state the preliminaries on the variational structure for (1). In the final section, the proof of Theorem 1 will be given via the $\mathbb{Z}_{2}$-genus theory, together with an approximating argument.

\section{Preliminaries}

Let $L^{2}\left(S^{1}, \mathbb{R}^{n}\right)$ denote the set of $n$-tuples of $2 \pi$-periodic functions which are square integrable. If $x \in L^{2}\left(S^{1}, \mathbb{R}^{n}\right)$, it has a Fourier expansion

$$
x(t)=a_{0}+\sum_{j \in \mathbb{N}}\left(a_{j} \cos j t+b_{j} \sin j t\right),
$$

where $a_{i}, b_{j} \in \mathbb{R}^{n}$ and the series converges in the space $L^{2}\left(S^{1}\right.$, $\left.\mathbb{R}^{n}\right)$. For $x \in L^{2}\left(S^{1}, \mathbb{R}^{n}\right)$ with its expansion (6), set $H:=\{x \in$ $\left.L^{2}\left(S^{1}, \mathbb{R}^{n}\right) \mid\|x\|_{H}<\infty\right\}$, where

$$
\|x\|_{H}:=\left|a_{0}\right|^{2}+\sum_{j \in \mathbb{N}}(1+j)\left(\left|a_{j}\right|^{2}+\left|b_{j}\right|^{2}\right) .
$$

Then $H$, equipped with the norm $\|\cdot\|_{H}$, is a Sobolev space.

On the other hand, for $x \in H$ with its expansion (6), set

$$
\|x\|:=\left|a_{0}\right|^{2}+\sum_{j \in \mathbb{N}} j\left(\left|a_{j}\right|^{2}+\left|b_{j}\right|^{2}\right) .
$$

Then $H$ possesses another norm $\|\cdot\|$ which is equivalent to $\|\cdot\|_{H}$. In the following, we always employ $\|\cdot\|$ as the norm of $H$. The associated inner product with $\|\cdot\|$ is denoted by $\langle\cdot, \cdot\rangle$.

Now set

$$
E:=\{x \in H \mid x(\cdot+\pi)=-x(\cdot)\} .
$$

Then $E$ is a closed subspace of $H$ and the Fourier expansion of $x \in E$ reduces to

$$
x(t)=\sum_{j=1}^{\infty}\left[a_{2 j-1} \cos (2 j-1) t+b_{2 j-1} \sin (2 j-1) t\right] .
$$

Thus with $x_{1}, x_{2} \in E$ being expanded as

$$
\begin{array}{r}
x_{i}(t)=\sum_{j=1}^{\infty}\left[a_{2 j-1}^{(i)} \cos (2 j-1) t+b_{2 j-1}^{(i)} \sin (2 j-1) t\right], \\
i=1,2,
\end{array}
$$

we have

$$
\left\langle x_{1}, x_{2}\right\rangle=\sum_{j=1}^{\infty}(2 j-1)\left\{\left(a_{2 j-1}^{(1)}, a_{2 j-1}^{(2)}\right)+\left(b_{2 j-1}^{(1)}, b_{2 j-1}^{(2)}\right)\right\} .
$$

For $x, y \in E$, we call $y$ a weak derivative of $x$ and denote it by $\dot{x}=y$ if

$$
\begin{array}{r}
\int_{0}^{2 \pi}\left(x(t), z^{\prime}(t)\right) d t=-\int_{0}^{2 \pi}(y(t), z(t)) d t \\
\forall z \in C^{\infty}\left(S^{1}, \mathbb{R}^{n}\right) .
\end{array}
$$

Further, for $x \in C^{\infty}\left(S^{1}, \mathbb{R}^{n}\right) \cap E$ with its expansion (10), define

$$
\begin{aligned}
A(x) & :=\frac{1}{2} \int_{0}^{2 \pi}\left(\dot{x}\left(t+\frac{\pi}{2}\right), x(t)\right) d t \\
& =\frac{1}{2} \sum_{j=1}^{\infty}(-1)^{j}(2 j-1)\left(\left|a_{2 j-1}\right|^{2}+\left|b_{2 j-1}\right|^{2}\right) .
\end{aligned}
$$


Then it is easy to check that $|A(x)| \leqslant\|x\|^{2}$ for $x \in C^{\infty}\left(S^{1}\right.$, $\left.\mathbb{R}^{n}\right) \cap E$. Therefore $A$ extends to all of $E$ as a continuous quadratic form. This extension will still be denoted by $A$.

Let $\widetilde{F} \in C^{1}\left(\mathbb{R}^{n}, \mathbb{R}\right)$ and satisfy

$$
\widetilde{F}(-x)=\widetilde{F}(x), \quad|\widetilde{F}(x)| \leqslant C_{1}+C_{2}|x|^{s}, \quad x \in \mathbb{R}^{n}
$$

for some $s \in[1, \infty)$. Define

$$
\Phi(x):=\int_{0}^{2 \pi} \widetilde{F}(x(t)) d t, \quad x \in E
$$

and $I(x)=A(x)+\Phi(x), x \in E$. The following lemma is derived from [9, Lemma 2.2].

Lemma 5 (see [9]). Let $\widetilde{F} \in C^{1}\left(\mathbb{R}^{n}, \mathbb{R}\right)$ and satisfy (15). Then $I \in C^{1}(E, \mathbb{R})$ and

$$
I^{\prime}(x) y=A^{\prime}(x) y+\int_{0}^{2 \pi}\left(\widetilde{F}_{x}(x(t)), y(t)\right), \quad y \in E,
$$

where

$$
A^{\prime}(x) y=\int_{0}^{2 \pi}\left(\dot{x}\left(t+\frac{\pi}{2}\right), y(t)\right) d t, \quad y \in E .
$$

Moreover, the existence of $2 \pi$-periodic solutions $x(t)$ for

$$
x^{\prime}(t)=-\widetilde{F}_{x}\left(x\left(t-\frac{\pi}{2}\right)\right)
$$

satisfying $x \in E$ is equivalent to the existence of critical points of functional $I$.

Let $\left\{e_{1}, \ldots, e_{n}\right\}$ be the orthonormal basis of $\mathbb{R}^{n}$. For $k \in \mathbb{N}$, set

$$
\begin{gathered}
E_{+}(k):=\operatorname{span}\left\{\cos [(4 k-1) t] e_{i}, \sin [(4 k-1) t] e_{i}:\right. \\
i=1,2, \ldots, n\},
\end{gathered}
$$$$
E_{-}(k):=\operatorname{span}\left\{\cos [(4 k-3) t] e_{i}, \sin [(4 k-3) t] e_{i}:\right.
$$$$
i=1,2, \ldots, n\} .
$$

For $l, m \in \mathbb{N} \cup\{+\infty\}$, define

$$
V_{l}^{ \pm}=\overline{\oplus_{k=1}^{l} E_{ \pm}(k)}, \quad V_{l}^{m}=V_{l}^{-} \oplus V_{m}^{+},
$$

where the closure is of $E$ sense. Set $V^{ \pm}:=V_{+\infty}^{ \pm}$; then $E=V^{+} \oplus$ $V^{-}$. In the rest of this paper, this decomposition will always be referred to when a point $x \in E$ is written as $x=x^{+}+x^{-}$, where $x^{ \pm} \in V^{ \pm}$.

Remark 6. In view of (12), (14), and (18), we see that

$$
A(x)=\frac{1}{2}\left(\left\|x^{+}\right\|^{2}-\left\|x^{-}\right\|^{2}\right)
$$

and that

$$
A^{\prime}(x) y=\left\langle x^{+}, y^{+}\right\rangle-\left\langle x^{-}, y^{-}\right\rangle, \quad x, y \in E .
$$

The following lemma is derived from [32, Lemma 2.1].

Lemma 7 (see [32]). For each $s \in[1, \infty)$ there is $\gamma_{s}>0$ such that

$$
\|x\|_{s} \leqslant \gamma_{s} m^{-1 / s}\|x\|
$$

for all $x \in\left(V_{m-1}^{m-1}\right)^{\perp}$ with $m \geqslant 2$, the orthogonal complement in $E$, where (and below) $\|\cdot\|_{s}$ denotes the usual $L^{s}$-norm.

\section{Proof of Theorem 1}

Without loss of generality we assume that $\sigma=\pi / 2$ since, under the change of variables $y(t)=x(2 \sigma t / \pi)$, (1) can be transformed into the system

$$
\dot{y}(t)=-\tilde{f}\left(y\left(t-\frac{\pi}{2}\right)\right),
$$

where $\tilde{f}(y)=(2 \sigma / \pi) f(y)$ still satisfies $\left(\mathrm{F}_{1}-\mathrm{F}_{3}\right)$ with $f$ being replaced by $\tilde{f}$.

Let $\chi \in C^{\infty}(\mathbb{R},[0,1])$ be such that $\chi(s)=0$ for $s \leqslant r_{0} / 2$, $\chi(s)=1$ for $s \geqslant r_{0}$, and $\chi^{\prime}(s)>0$ for $s \in\left(r_{0} / 2, r_{0}\right)$. Define $\widetilde{F}: \quad \mathbb{R}^{n} \mapsto \mathbb{R}$ by

$$
\widetilde{F}(x):=(1-\chi(|x|)) F(x)+\chi(|x|) M_{0}|x|^{\alpha},
$$

where $M_{0}=\inf \left\{F(x) / r_{0}^{\alpha}: r_{0} / 2 \leqslant|x| \leqslant r_{0}\right\}$.

Let $\alpha^{\prime}>0$ be such that $1 / \alpha+1 / \alpha^{\prime}=1$. By $\left(\mathrm{F}_{3}\right)$ we get

$$
\begin{gathered}
0<\left(\widetilde{F}_{x}(x), x\right) \leqslant \alpha \widetilde{F}(x), \quad \forall x \in \mathbb{R}^{n}, \\
\widetilde{F}(x) \geqslant \begin{cases}C_{1}\left|\widetilde{F}_{x}(x)\right|^{\beta^{\prime}}, & |x| \leqslant 1, \\
C_{1}\left|\widetilde{F}_{x}(x)\right|^{\alpha^{\prime}}, & |x|>1,\end{cases}
\end{gathered}
$$

where (and below) $C_{j}$ 's stand for positive constants.

Lemma 8. Let $\widetilde{F}: \mathbb{R}^{n} \mapsto \mathbb{R}$ be defined by (25); then $1<\beta<$ $\alpha<2, \widetilde{F} \in C^{1}\left(\mathbb{R}^{n}, \mathbb{R}\right)$, and

$$
C_{2}|x|^{\alpha} \leqslant \widetilde{F}(x) \leqslant \begin{cases}C_{3}|x|^{\beta}, & |x| \leqslant 1, \\ C_{3}|x|^{\alpha}, & |x|>1 .\end{cases}
$$

Proof. From (25), it is easy to see that $\widetilde{F} \in C^{1}\left(\mathbb{R}^{n}, \mathbb{R}\right)$. Now we start to prove (28). Let $M$ be such a constant that $\mid \ln \widetilde{F}(x)-$ $\alpha \ln |x| \mid \leqslant M$ for $x \in S_{1} \equiv \partial B_{1}$. For $x \in \mathbb{R}^{n},|x| \leqslant 1$, set $x_{0}=x /|x|$; then $x_{0} \in S_{1}$. Define $g(t)=\ln \widetilde{F}\left(t x_{0}\right)-\alpha \ln \left|t x_{0}\right|$, $t \in(0,1]$. Then, by (26),

$$
g^{\prime}(t)=\left(\frac{\widetilde{F}_{x}\left(t x_{0}\right)}{\widetilde{F}\left(t x_{0}\right)}, x_{0}\right)-\alpha \leqslant 0,
$$

which implies that $g(|x|) \geqslant g(1)$; that is,

$$
\ln \widetilde{F}(x)-\alpha \ln |x| \geqslant \ln \widetilde{F}\left(x_{0}\right)-\alpha \ln \left|x_{0}\right| \geqslant-M .
$$

It follows that $\widetilde{F}(x) \geqslant e^{-M}|x|^{\alpha}$ for $|x| \leqslant 1$, which, combining with (25), leads to the inequality on the left hand of (28) with $\mathrm{C}_{2}$ being chosen adequately. 
Again, for $x \in \mathbb{R}^{n},|x| \leqslant 1$, set $x_{0}=x /|x|$ and define $h(t)=\left(\widetilde{F}\left(t x_{0}\right)\right)^{1 / \beta}-t\left|x_{0}\right| /\left(\beta C_{1}^{1 / \beta^{\prime}}\right), t \in[0,1]$. Then by the first inequality in (27),

$$
\begin{aligned}
h^{\prime}(t) & =\frac{1}{\beta}\left(\frac{\left(\widetilde{F}_{x}\left(t x_{0}\right), x_{0}\right)}{\left(\widetilde{F}\left(t x_{0}\right)\right)^{1 / \beta^{\prime}}}-\frac{1}{C_{1}^{1 / \beta^{\prime}}}\left|x_{0}\right|\right) \\
& \leqslant \frac{\left|x_{0}\right|}{\beta}\left(\frac{\left|\widetilde{F}_{x}\left(t x_{0}\right)\right|}{\left(\widetilde{F}\left(t x_{0}\right)\right)^{1 / \beta^{\prime}}}-\frac{1}{C_{1}^{1 / \beta^{\prime}}}\right)<0 .
\end{aligned}
$$

Thus $h(|x|) \leqslant h(0)=0$, which leads to $\widetilde{F}(x) \leqslant C_{3}^{\prime}|x|^{\beta}$, where $C_{3}^{\prime}=1 /\left(\beta C_{1}^{1 / \beta^{\prime}}\right)^{\beta}$. In the same way, from the second inequality in (27), we can arrive at $\widetilde{F}(x) \leqslant C_{3}^{\prime \prime}|x|^{\alpha}$ for $|x|>$ 1 , where the constant $C_{3}^{\prime \prime}$ only depends on $\alpha$ and $C_{1}$. With $C_{3}=\max \left\{C_{3}^{\prime}, C_{3}^{\prime \prime}\right\}$, the inequalities on the right hand of (28) hold. Thus we get (28), which implies that $C_{2}|x|^{\alpha} \leqslant C_{3}|x|^{\beta}$ for $|x| \leqslant 1$ and that $1<\beta<\alpha<2$. The proof is complete.

Now we consider the functional

$$
I(x)=A(x)+\int_{0}^{2 \pi} \widetilde{F}(x) d t, \quad x \in E .
$$

Lemma 9. I satisfies (PS) condition; that is, every sequence $\left\{x_{k}\right\} \subset E$ such that $\left\{I\left(x_{k}\right)\right\}$ is bounded and $I^{\prime}\left(x_{k}\right) \rightarrow 0$ as $k \rightarrow \infty$ has a convergent subsequence.

Proof. By Lemma 5, for $x \in E, I^{\prime}(x)$ is defined by

$$
I^{\prime}(x) y=A^{\prime}(x) y+\int_{0}^{2 \pi}\left(\widetilde{F}_{x}(x), y\right) d t, \quad \forall y \in E .
$$

To verify that $I$ satisfies (PS) condition, we suppose $\left|I\left(x_{k}\right)\right| \leqslant$ $C_{4}$ and $I^{\prime}\left(x_{k}\right) \rightarrow 0$ as $k \rightarrow \infty$. Note that, for large $k$, $\left|I^{\prime}\left(x_{k}\right) x\right| \leqslant\|x\|$. Thus for large $k$ and $x=x_{k}$, from (32) and (33),

$$
\begin{aligned}
C_{4}+\|x\| & \geqslant I(x)-\frac{1}{2} I^{\prime}(x) x \\
& =\int_{0}^{2 \pi}\left[\widetilde{F}(x)-\frac{1}{2}\left(\widetilde{F}_{x}(x), x\right)\right] d t .
\end{aligned}
$$

Noticing that $1<\beta<\alpha<2$, we see from (25) that, for all $x \in \mathbb{R}^{n}$,

$$
\left(\widetilde{F}_{x}(x), x\right) \geqslant C_{5} \max \left\{|x|^{\alpha},|x|^{\beta}\right\}-C_{6},
$$

which, combining with (26) and (34), implies

$$
\begin{gathered}
C_{4}+\|x\| \geqslant\left(\alpha^{-1}-2^{-1}\right) \int_{0}^{2 \pi}\left(\widetilde{F}_{x}(x), x\right) d t \\
\geqslant C_{7} \max \left\{\|x\|_{\alpha}^{\alpha},\|x\|_{\beta}^{\beta}\right\}-C_{8}, \\
\max \left\{\|x\|_{\alpha}^{\alpha},\|x\|_{\beta}^{\beta}\right\} \leqslant C_{9}(\|x\|+1) .
\end{gathered}
$$

Next for large $k$, taking $x=x_{k}$ and $\varsigma=x_{k}^{+}$in

$$
\left|\int_{0}^{2 \pi}\left(\widetilde{F}_{x}(x), \varsigma\right) d t+A^{\prime}(x) \varsigma\right|=\left|I^{\prime}(x) \varsigma\right| \leqslant\|\varsigma\|
$$

and using (23), (27), and (28) and the Hölder inequality $(1 / \alpha+$ $\left.1 / \alpha^{\prime}=1,1 / \beta+1 / \beta^{\prime}=1\right)$, we get

$$
\begin{aligned}
\left\|x^{+}\right\|^{2} \leqslant & \left|\int_{0}^{2 \pi}\left(\widetilde{F}_{x}(x), x^{+}\right) d t\right|+\left\|x^{+}\right\| \\
\leqslant & C_{10}\left(\int_{|x(t)|>1}|x|^{\alpha / \alpha^{\prime}}\left|x^{+}\right| d t+\int_{|x(t)| \leqslant 1}|x|^{\beta / \beta^{\prime}}\left|x^{+}\right| d t\right) \\
& +\left\|x^{+}\right\| \\
\leqslant & C_{10}\left(\|x\|_{\alpha}^{\alpha / \alpha^{\prime}}\left\|x^{+}\right\|_{\alpha}+\|x\|_{\beta}^{\beta / \beta^{\prime}}\left\|x^{+}\right\|_{\beta}\right)+\left\|x^{+}\right\| \\
\leqslant & C_{11}\left(\|x\|_{\alpha}^{\alpha / \alpha^{\prime}}+\|x\|_{\beta}^{\beta / \beta^{\prime}}+1\right)\left\|x^{+}\right\|,
\end{aligned}
$$

where the last inequality holds since $E$ is compactly embedded in $L^{s}\left(S^{1}, \mathbb{R}^{n}\right)$ for $s \geqslant 1$. It follows from (36) that

$$
\left\|x^{+}\right\| \leqslant C_{12}\left(\|x\|^{1 / \alpha^{\prime}}+\|x\|^{1 / \beta^{\prime}}+1\right) .
$$

Similarly, (40) works with $x^{+}$being replaced by $x^{-}$. Combining these inequalities shows

$$
\|x\| \leqslant C_{13}\left(\|x\|^{1 / \alpha^{\prime}}+\|x\|^{1 / \beta^{\prime}}+1\right),
$$

which implies that $\left\{x_{k}\right\}$ is bounded in $E$.

Let $\Phi$ be defined by (16). By [33, Proposition B.37], $\left\{\Phi^{\prime}\left(x_{k}\right)\right\}$ is precompact in E. Moreover, from (23) and (33),

$$
I^{\prime}\left(x_{k}\right)=x_{k}^{+}-x_{k}^{-}+\Phi^{\prime}\left(x_{k}\right) .
$$

It follows that $\left\{x_{k}\right\}$ has a convergent subsequence. The proof is complete.

Lemma 10. For each $l \in \mathbb{N}$, there are $\rho_{l}>0, a_{l}>0$, and $0<b_{l} \rightarrow 0$ such that
(a) $I(x) \geqslant 0$, for all $x \in B_{\rho_{l}} \cap V_{l}^{+\infty}$ and $\inf I\left(\partial S_{\rho_{l}} \cap V_{l}^{+\infty}\right) \geqslant$ $a_{l}$, where $B_{\rho_{l}}=\left\{x \in E:\|x\| \leqslant \rho_{l}\right\}$;
(b) $\sup I\left(\left(V_{l-1}^{+\infty}\right)^{\perp}\right) \leqslant b_{l}$.

Proof. Noticing that $V_{l}^{+\infty}=V_{l}^{-} \oplus V_{+\infty}^{+}$and that $\operatorname{dim}\left(V_{l}^{-}\right)<$ $\infty$, we have, for $x \in B_{\rho_{l}} \cap V_{l}^{+\infty}$,

$$
\begin{aligned}
\|x\|_{\alpha} & =\sup \left\{\int_{S^{1}}(x(t), y(t)) d t \mid y \in L^{\alpha^{\prime}}\left(S^{1}, \mathbb{R}^{n}\right),\|y\|_{\alpha^{\prime}}=1\right\} \\
& \geqslant \sup \left\{\int_{S^{1}}(x(t), y(t)) d t \mid y \in V_{l}^{-},\|y\|_{\alpha^{\prime}}=1\right\} \\
& =\sup \left\{\int_{S^{1}}\left(x^{-}(t), y(t)\right) d t \mid y \in V_{l}^{-},\|y\|_{\alpha^{\prime}}=1\right\} \\
& =\left\|x^{-}\right\|_{\alpha} \geqslant \eta_{l}\left\|x^{-}\right\|,
\end{aligned}
$$


where $\eta_{l}$ is a positive constant depending on $l$. It follows by (28) that, for $x \in B_{\rho_{l}} \cap V_{l}^{+\infty}$,

$$
\begin{aligned}
I(x) & \geqslant \frac{1}{2}\left\|x^{+}\right\|^{2}+C_{2} \eta_{l}\left\|x^{-}\right\|^{\alpha}-\frac{1}{2}\left\|x^{-}\right\|^{2} \\
& =\frac{1}{2}\left\|x^{+}\right\|^{2}+\left(C_{2} \eta_{l}-\frac{1}{2}\left\|x^{-}\right\|^{2-\alpha}\right)\left\|x^{-}\right\|^{\alpha},
\end{aligned}
$$

which implies (a) by setting $\rho_{l}:=\min \left\{1,\left(C_{2} \eta_{l}\right)^{1 /(2-\alpha)}\right\}$ and $a_{l}=\rho_{l}^{2}\left(1+C_{2} \eta_{l}\right) / 2$.

Let $x \in\left(V_{l-1}^{+\infty}\right)^{\perp}$. By $(28)$ and Lemma 7,

$$
\begin{aligned}
I(x) & \leqslant C_{3}\left(\|x\|_{\alpha}^{\alpha}+\|x\|_{\beta}^{\beta}\right)-\frac{1}{2}\|x\|^{2} \\
& \leqslant C_{16} l^{-1}\left(\|x\|^{\alpha}+\|x\|^{\beta}\right)-\frac{1}{2}\|x\|^{2} \\
& \leqslant b_{l}:=\sup _{s \geq 0} g(s),
\end{aligned}
$$

where $g(s):=C_{16} l^{-1}\left(s^{\alpha}+s^{\beta}\right)-s^{2} / 2$. Noticing $1<\beta \leqslant \alpha<2$, one can see that $b_{l} \rightarrow 0$ as $l \rightarrow \infty$ and (b) follows. The proof is complete.

In the following, let $\Sigma$ denote the family of closed (in $E$ ) subsets of $E \backslash\{\mathbf{0}\}$ symmetric with respect to the origin, and

$$
\gamma: \Sigma \longmapsto \mathbb{N} \cup\{0, \infty\}
$$

the $\mathbb{Z}_{2}$-genus map (see [33]). For $l, m \in \mathbb{N}$, set

$$
\Sigma_{l}^{m}=\left\{A \in \Sigma: A \subset V_{+\infty}^{m}, \gamma(A) \geqslant n(l+m)\right\},
$$

and define

$$
c_{l, m}=\sup _{A \in \Sigma_{l}^{m}} \inf _{x \in A} I(u)
$$

Lemma 11. For all $l, m \in \mathbb{N}, c_{l, m}$ is a critical value of $I$ and

$$
a_{l} \leqslant c_{l, m} \leqslant b_{l} .
$$

Proof. We first prove that (49) holds. For each $m \in \mathbb{N}$, let $\rho_{l}$ be chosen as that in Lemma 10; then it follows by Lemma 10(a) that inf $I\left(\partial S_{\rho_{l}} \cap V_{l}^{+\infty}\right) \geqslant a_{l}$. Denote $\widetilde{A}=\partial S_{\rho_{l}} \cap V_{l}^{m}$; then $\gamma(\widetilde{A})=n(m+l)$ and $\widetilde{A} \in \Sigma_{l}^{m}$. Since $\widetilde{A} \subset \partial S_{\rho_{l}} \cap V_{l}^{+\infty}$, we have

$$
c_{l, m} \geqslant \inf _{x \in \widetilde{A}} I(x) \geqslant \inf I\left(\partial S_{\rho_{l}} \cap V_{l}^{+\infty}\right) \geqslant a_{l} .
$$

On the other hand, for every $A \in \Sigma_{l}^{m}$, by the property of genus, $A \cap\left(V_{l-1}^{+\infty}\right)^{\perp} \neq \varnothing$, which, from Lemma 10(b), leads to $\inf _{x \in A} I(x) \leqslant b_{l}$ for every $A \in \Sigma_{l}^{m}$. Thus $c_{l, m} \leqslant b_{l}$ and (49) holds.

By $\left(\mathrm{F}_{1}\right)$ and $(25), \widetilde{F}(x)$ is even with respect to $x$, which implies that $I$ is even. We claim that $c=c_{l, m}$ is a critical point of $I$. Otherwise, there exists $\epsilon>0$, such that there is no any critical point in the interval $(c-\epsilon, c+\epsilon)$. By the definition of $c_{l, m}$, there exists $A \in \Sigma_{l}^{m}$, such that

$$
\inf _{x \in A} I(x)>c-\epsilon .
$$

For $a \in \mathbb{R}$, denote $I^{a}=\{x \in E: I(x) \geqslant a\}$. Use a positive rather than a negative gradient flow [33, Remark A.17], we get $\eta \in C([0,1] \times E, E)$ such that $\eta(1, \cdot)$ is odd and

$$
\eta\left(1, I^{c-\epsilon}\right) \subset I^{c+\epsilon}
$$

Since $A \subset I^{c-\epsilon}$, we have $\eta(1, A) \subset I^{c+\epsilon}$; that is,

$$
\inf _{x \in \eta(1, A)} I(x) \geqslant c+\epsilon .
$$

On the other hand, by the property of genus, we know that $\gamma(\eta(1, A)) \in \Sigma_{l}^{m}$, which, by the definition of $c$, leads to

$$
c \geqslant \inf _{x \in \eta(1, A)} I(x) \geqslant c+\epsilon .
$$

This contradiction implies that $c_{l, m}$ is a critical value of $I$. The proof is complete.

Now we are in a position to give the following proof.

Proof of Theorem 1. In view of Lemma 11, let $x_{l, m} \in V_{+\infty}^{m}$ be such that

$$
I\left(x_{l, m}\right)=c_{l, m}, \quad I^{\prime}\left(x_{l, m}\right)=0 .
$$

Then by (PS) condition, along a subsequence as $m \rightarrow \infty$, $x_{l, m} \rightarrow x_{l} \in E$ such that

$$
a_{l} \leqslant I\left(x_{l}\right) \leqslant b_{l}, \quad I^{\prime}\left(x_{l}\right)=0,
$$

which implies that $x_{l}$ is nonzero. Moreover, by Lemma 5,

$$
x_{l}^{\prime}(t)=-\widetilde{F}_{x}\left(x_{l}\left(t-\frac{\pi}{2}\right)\right) .
$$

We claim that, for sufficiently large $l, x_{l}$ solves (1). In fact, from (26) and (56)

$$
\begin{aligned}
b_{l} & \geqslant I\left(x_{l}\right)=I\left(x_{l}\right)-\frac{1}{2} I^{\prime}\left(x_{l}\right) x_{l} \\
& \geqslant\left(1-\frac{\alpha}{2}\right) \int_{0}^{2 \pi} \widetilde{F}\left(x_{l}\right) d t .
\end{aligned}
$$

By (27), (58), and Hölder inequality

$$
\begin{aligned}
\left\|x_{l}^{+}\right\|^{2}= & \int_{0}^{2 \pi}\left(\widetilde{F}_{x}\left(x_{l}\right), x_{l}^{+}\right) d t \\
\leqslant & \left\|x_{l}^{+}\right\|_{\alpha}\left(\int_{0}^{2 \pi}\left|\widetilde{F}_{x}\left(x_{l}\right)\right|^{\alpha^{\prime}} d t\right)^{1 / \alpha^{\prime}} \\
& +\left\|x_{l}^{+}\right\|_{\beta}\left(\int_{0}^{2 \pi}\left|\widetilde{F}_{x}\left(x_{l}\right)\right|^{\beta^{\prime}} d t\right)^{1 / \beta^{\prime}} \\
\leqslant & C_{17}\left\|x_{l}^{+}\right\|\left(\int_{0}^{2 \pi} \widetilde{F}\left(x_{l}\right) d t\right)^{1 / \alpha^{\prime}} \\
& +C_{18}\left\|x_{l}^{+}\right\|\left(\int_{0}^{2 \pi} \widetilde{F}\left(x_{l}\right) d t\right)^{1 / \beta^{\prime}} \\
\leqslant & \left(C_{17} b_{l}^{1 / \alpha^{\prime}}+C_{18} b_{l}^{1 / \beta^{\prime}}\right)\left\|x^{+}\right\| .
\end{aligned}
$$


Similarly, the above inequality works with $x_{l}^{+}$replaced by $x_{l}^{-}$. These inequalities yield

$$
\left\|x_{l}\right\| \leq C_{19} b_{l}^{1 / \alpha^{\prime}}+C_{20} b_{l}^{1 / \beta^{\prime}} .
$$

Since $b_{l} \rightarrow 0$ as $l \rightarrow \infty$, it follows that

$$
\left\|x_{l}\right\| \longrightarrow 0 \quad \text { as } l \longrightarrow \infty \text {. }
$$

Furthermore, from (27) and (57), we have

$$
\begin{aligned}
\int_{0}^{2 \pi}\left|\dot{x}_{l}(t)\right|^{2} d t & =\int_{0}^{2 \pi}\left|\widetilde{F}_{x}\left(x_{l}\right)\right|^{2} d t \\
& \leqslant C_{21}\left(\int_{0}^{2 \pi}\left[\widetilde{F}\left(x_{l}\right)\right]^{2 / \beta^{\prime}} d t+\int_{0}^{2 \pi}\left[\widetilde{F}\left(x_{l}\right)\right]^{2 / \alpha^{\prime}} d t\right) .
\end{aligned}
$$

It follows from (58) that $\left\|\dot{x}_{l}\right\|_{2} \rightarrow 0$ as $l \rightarrow \infty$. Recalling (61), we get

$$
\left\|x_{l}\right\|_{W^{1,2}} \longrightarrow 0 \quad \text { as } l \longrightarrow \infty
$$

which implies that $\left\|x_{l}\right\|_{\infty} \rightarrow 0$ as $l \rightarrow 0$. Thus for $m$ sufficiently large, $\left\|x_{l}\right\|_{\infty}<r_{0} / 2$ and therefor $\widetilde{F}_{x}\left(x_{l}\right)=F_{x}\left(x_{l}\right)$. It follow from (57) that, for $l$ sufficiently large, $x_{l}$ solves (1). In addition, by (1) and $\left(\mathrm{F}_{3}\right)(\mathrm{i})$, the only constant solution of (1) is the trivial solution. Then (56) yields that $x_{l}$ is nonconstant and the proof of Theorem 1 is complete.

\section{Acknowledgments}

The authors are grateful to the referees' careful reviewing and helpful comments. The work was supported by BJJW (KZ201310028031 and KM2014).

\section{References}

[1] X.-L. Liu and W.-T. Li, "Existence and uniqueness of positive periodic solutions of functional differential equations," Journal of Mathematical Analysis and Applications, vol. 293, no. 1, pp. 28-39, 2004.

[2] S. Padhi and S. Srivastava, "Existence of three periodic solutions for a nonlinear first order functional differential equation," Journal of the Franklin Institute, vol. 346, no. 8, pp. 818-829, 2009.

[3] R. Ma, R. Chen, and T. Chen, "Existence of positive periodic solutions of nonlinear first-order delayed differential equations," Journal of Mathematical Analysis and Applications, vol. 384, no. 2, pp. 527-535, 2011.

[4] R. Olach, "Positive periodic solutions of delay differential equations," Applied Mathematics Letters, vol. 26, pp. 1141-1145, 2013.

[5] P. Amster and L. Idels, "Periodic solutions in general scalar nonautonomous models with delays," Nonlinear Differential Equations and Applications, vol. 20, no. 5, pp. 1577-1596, 2013.

[6] F. Giannakopoulos and A. Zapp, "Local and global Hopf bifurcation in a scalar delay differential equation," Journal of Mathematical Analysis and Applications, vol. 237, no. 2, pp. 425450, 1999.
[7] Y. Song and J. Wei, "Local Hopf bifurcation and global periodic solutions in a delayed predator-prey system," Journal of Mathematical Analysis and Applications, vol. 301, no. 1, pp. 1-21, 2005.

[8] S. Yuan and Y. Song, "Stability and Hopf bifurcations in a delayed Leslie-Gower predator-prey system," Journal of Mathematical Analysis and Applications, vol. 355, no. 1, pp. 82-100, 2009.

[9] Z. Guo and J. Yu, "Multiplicity results for periodic solutions to delay differential equations via critical point theory," Journal of Differential Equations, vol. 218, no. 1, pp. 15-35, 2005.

[10] X. Zhang and Q. Meng, "Nontrivial periodic solutions for delay differential systems via Morse theory," Nonlinear Analysis: Theory, Methods \& Applications A, vol. 74, no. 5, pp. 1960-1968, 2011.

[11] K. Wu and X. Wu, "Existence of periodic solutions for a class of first order delay differential equations dealing with vectors," Nonlinear Analysis: Theory, Methods \& Applications A, vol. 72, no. 12, pp. 4518-4529, 2010.

[12] C. Guo and Z. Guo, "Existence of multiple periodic solutions for a class of second-order delay differential equations," Nonlinear Analysis: Real World Applications, vol. 10, no. 5, pp. 3285-3297, 2009.

[13] J. Li, Z. Liu, and X. He, "Periodic solutions of some differential delay equations created by Hamiltonian systems," Bulletin of the Australian Mathematical Society, vol. 60, no. 3, pp. 377-390, 1999.

[14] Y.-T. Xu and Z.-M. Guo, "Applications of a $Z_{p}$ index theory to periodic solutions for a class of functional differential equations," Journal of Mathematical Analysis and Applications, vol. 257, no. 1, pp. 189-205, 2001.

[15] R. Hakl, A. Lomtatidze, and J. Sremr, "On a boundary-value problem of periodic type for first-order linear functional differential equations," Nonlinear Oscillations, vol. 5, no. 3, pp. 408425, 2002.

[16] R. Hakl, A. Lomtatidze, and J. Sremr, Some Boundary Value Problems for First Order Scalar Functional Dierential Equations, vol. 10 of Mathematica, Faculty of Science of the Masaryk University in Brno, Masaryk University, Brno, Czech Republic, 2002.

[17] A. Domoshnitsky, "Maximum principles and nonoscillation intervals for first order Volterra functional differential equations," Dynamics of Continuous, Discrete \& Impulsive Systems A, vol. 15, no. 6, pp. 769-814, 2008.

[18] I. Kiguradze and B. Puza, "On boundary value problems for systems of linear functional-differential equations," Czechoslovak Mathematical Journal, vol. 47, no. 2, pp. 341-373, 1997.

[19] I. Kiguradze and B. Puza, Boundary Value Problems for Systems of Linear Functional Differential Equations, FOLIA, Brno, Czech Republic, 2002.

[20] A. Domoshnitsky, R. Hakl, and J. Šremr, "Component-wise positivity of solutions to periodic boundary problem for linear functional differential system," Journal of Inequalities and Applications, vol. 2012, article 112, 2012.

[21] R. P. Agarwal, L. Berezansky, E. Braverman, and A. Domoshnitsky, Non-Oscillation Theory of Functional Differential Equations with Applications, Springer, New York, NY, USA, 2012.

[22] J. R. Graef and L. Kong, "Existence of multiple periodic solutions for first order functional differential equations," Mathematical and Computer Modelling, vol. 54, no. 11-12, pp. 29622968, 2011. 
[23] O. Diekmann, S. A. van Gils, S. M. V. Lunel, and H.-O. Walther, Delay-Equations: Functional Complex and Nonlinear Analysis, Springer, New York, NY, USA, 1995.

[24] W. Ge, "Existence of exactly $n+1$ simple 4-periodic solutions of the differential delay equation $\dot{x}(t)=-f(x(t-1))$," Acta Mathematica Sinica, vol. 10, no. 1, pp. 80-87, 1994.

[25] J. A. Yorke, "Periods of periodic solutions and the Lipschitz constant," Proceedings of the American Mathematical Society, vol. 22, pp. 509-512, 1969.

[26] J. Mawhin and W. Walter, "A general symmetry principle and some implications," Journal of Mathematical Analysis and Applications, vol. 186, no. 3, pp. 778-798, 1994.

[27] A. A. Zevin and M. A. Pinsky, "Minimal periods of periodic solutions of some Lipschitzian differential equations," Applied Mathematics Letters, vol. 22, no. 10, pp. 1562-1566, 2009.

[28] E. Bravyi, "About minimal periods of solutions of functional differential equations of the high orders," Izvestiya VUZov, Mathematics, vol. 12, pp. 72-77, 2013.

[29] A. Lasota and J. A. Yorke, "Bounds for periodic solutions of differential equations in Banach spaces," Journal of Differential Equations, vol. 10, pp. 83-91, 1971.

[30] T. Y. Li, "Bounds for the periods of periodic solutions of differential delay equations," Journal of Mathematical Analysis and Applications, vol. 49, pp. 124-129, 1975.

[31] M. Medved, "On minimal periods of functional-differential equations and difference inclusions," Annales Polonici Mathematici, vol. 3, pp. 263-270, 1991.

[32] Y. Ding and C. Lee, "Periodic solutions of Hamiltonian systems," SIAM Journal on Mathematical Analysis, vol. 32, no. 3, pp. 555571, 2000.

[33] P. H. Rabinowitz, Minimax Methods in Critical Point Theory with Applications to Differential Equations, vol. 65 of CBMS, American Mathematical Society, 1986. 


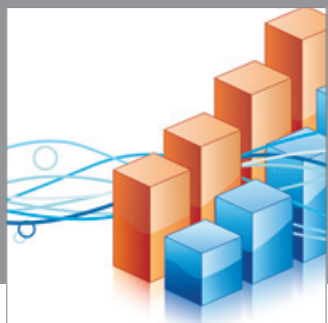

Advances in

Operations Research

mansans

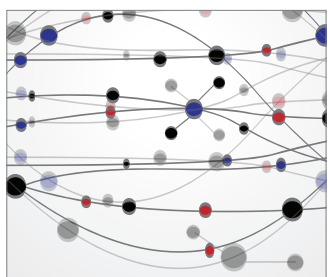

The Scientific World Journal
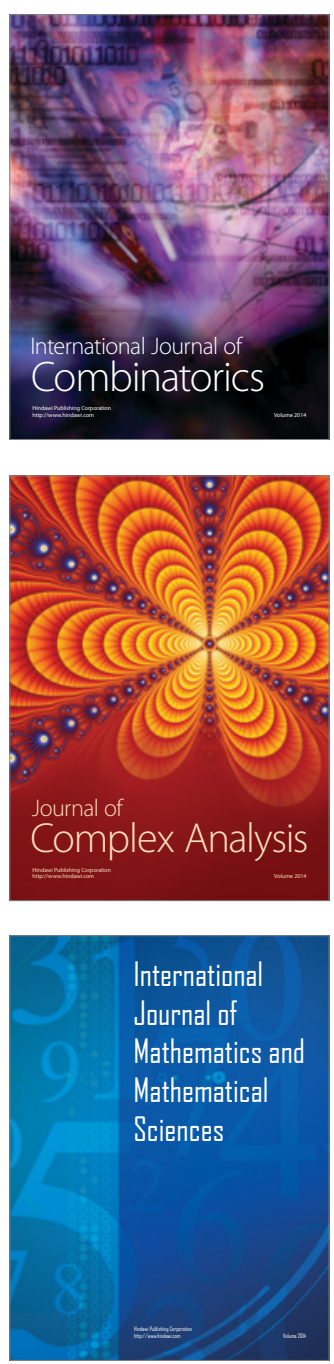
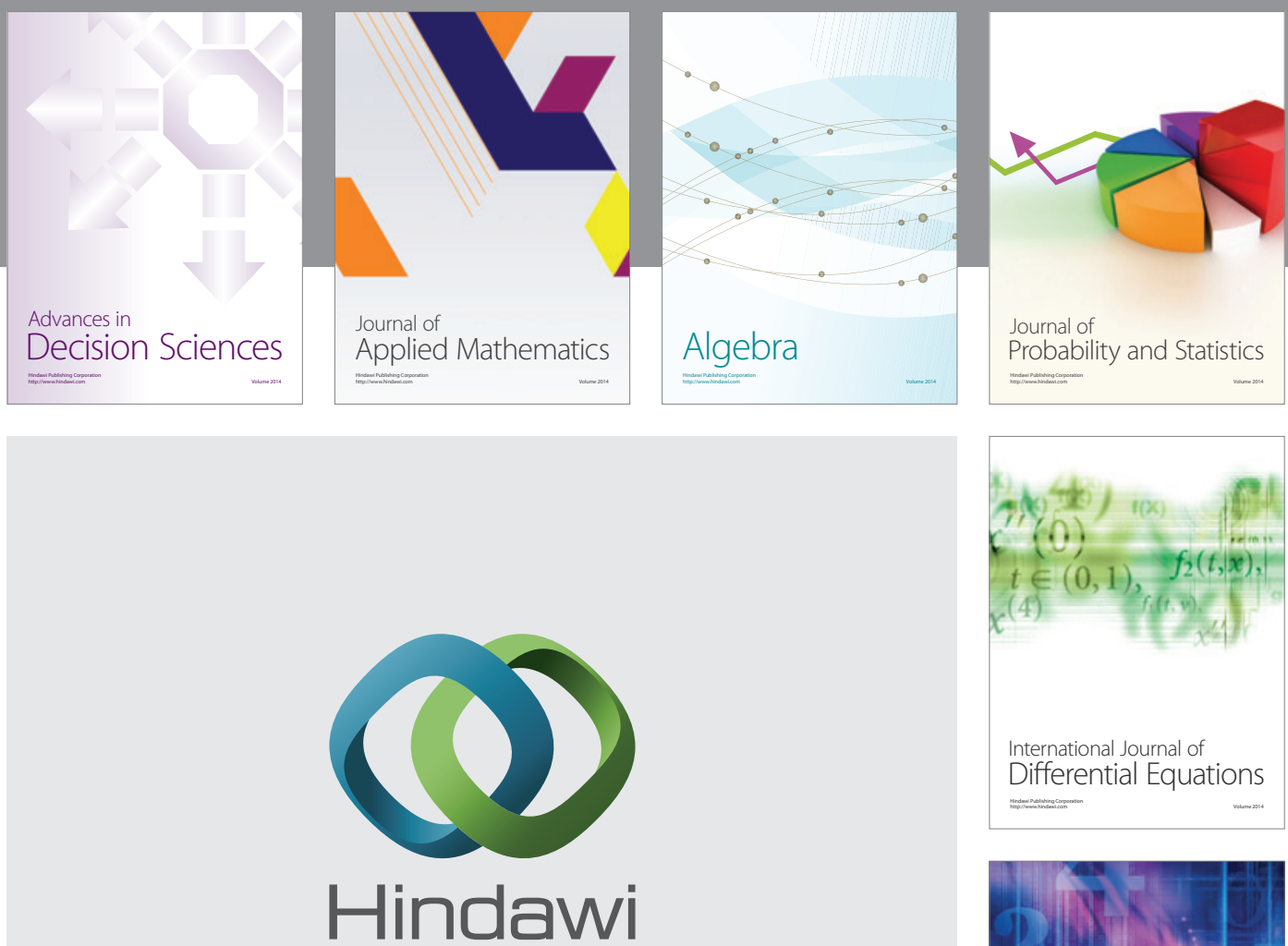

Submit your manuscripts at http://www.hindawi.com
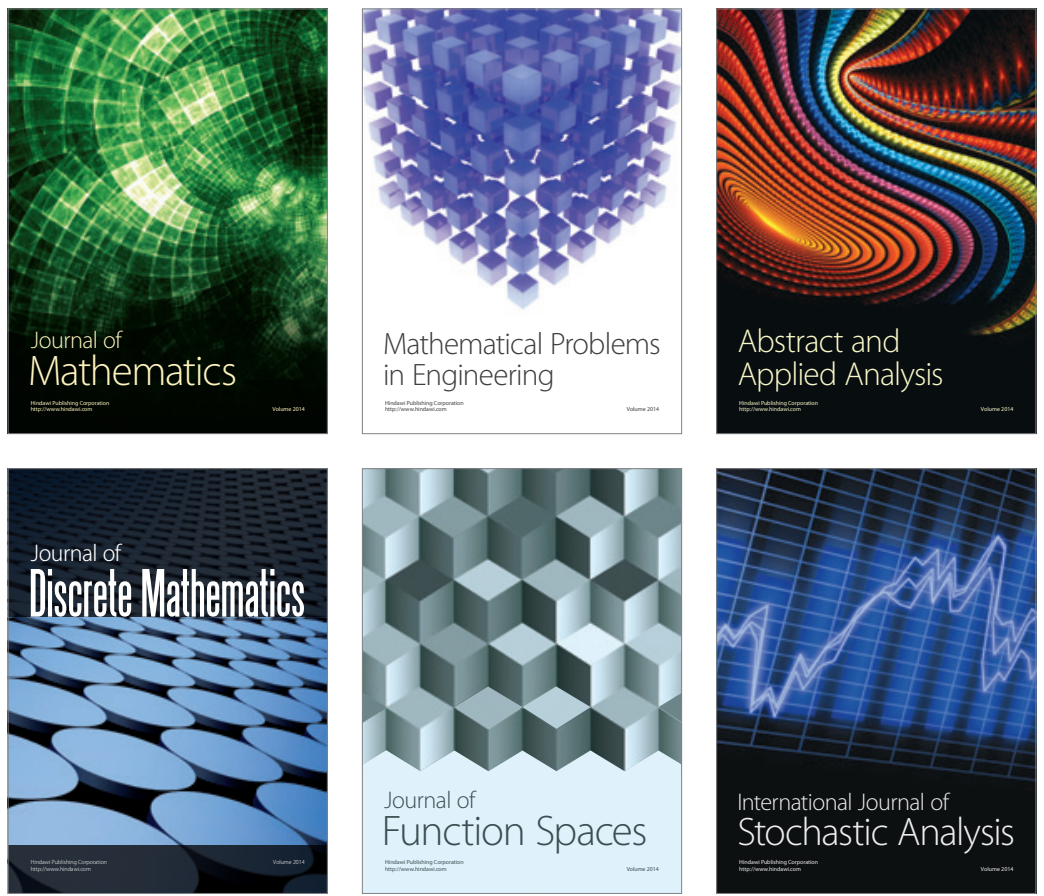

Journal of

Function Spaces

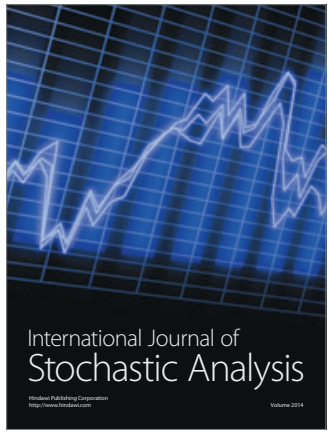

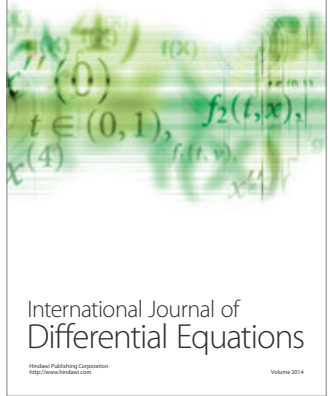
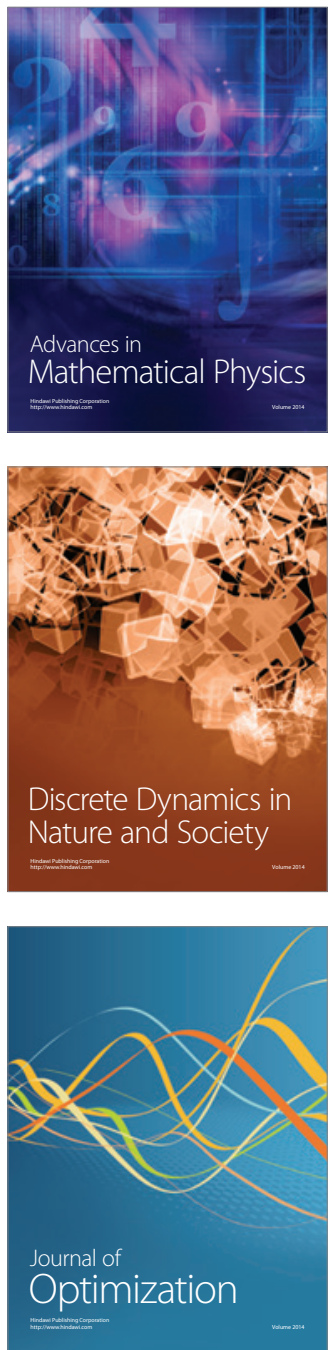